\title{
Origins of specificity during tDCS: anatomical, activity-selective, and input-bias mechanisms
}

\section{Marom Bikson* and Asif Rahman}

Department of Biomedical Engineering, The City College of the City University of New York, New York, NY, USA

Edited by:

Shennan Aibel Weiss, Albert Einstein

College of Medicine, USA

\section{Reviewed by:}

Lisa Marshall, University of Luebeck, Germany

Flavio Frohlich, University of North Carolina at Chapel Hill, USA

\section{${ }^{*}$ Correspondence:}

Marom Bikson, Department of Biomedical Engineering, The City College of the City University of New York, 160 Convent Avenue, New York, NY 10031, USA

email: bikson@ccny.cuny.edu
Transcranial Direct Current Stimulation (tDCS) is investigated for a broad range of neuropsychiatric indications, various rehabilitation applications, and to modulate cognitive performance in diverse tasks. Specificity of tDCS refers broadly to the ability of tDCS to produce precise, as opposed to diffuse, changes in brain function. Practically, specificity of tDCS implies application-specific customization of protocols to maximize desired outcomes and minimize undesired effects. Especially given the simplicity of tDCS and the complexity of brain function, understanding the mechanisms leading to specificity is fundamental to the rational advancement of tDCS. We define the origins of specificity based on anatomical and functional factors. Anatomical specificity derives from guiding current to targeted brain structures. Functional specificity may derive from either activity-selectivity, where active neuronal networks are preferentially modulated by tDCS, or input-selectivity, where bias is applied to different synaptic inputs. Rational advancement of tDCS may require leveraging all forms of specificity.

Keywords: transcranial direct current stimulation, anatomical brain connectivity, neuromodulation, transcranial magnetic stimulation, stimulation protocol

\section{THE NEED FOR tDCS SPECIFICITY}

As tDCS is a simple and general technique, applied to a wide range of clinical and cognitive neuroscience applications (Brunoni et al., 2012), a pivotal question to the rational advancement of tDCS is how is specificity achieved? More generally, why does low-intensity direct current produce (desirable) cognitive changes on highly complex tasks recruiting multiple neural pathways or treat multifarious neuropsychiatric disorders (Turkeltaub et al., 2012; Medina etal., 2013; Zimerman et al., 2013)? A question compounded when considering that the DC waveform is does not carry apparent information and that the induced electric field in the brain is low $[<1 \mathrm{~V} / \mathrm{m}$; (Datta etal., 2009; Ruffini et al., 2012)] producing minimal cell membrane polarization $[<1 \mathrm{mV}$; (Radman et al., 2009)]? Practically, how can stimulation protocols be optimized to promote specificity with the goal of increasing efficacy while reducing undesired side effects? Since these issues are central for the rational advancement of $\mathrm{tDCS}$, here we define both anatomical and functional origins of specificity (Cano et al., 2013). Although task specific effects of tDCS have been shown (Saucedo Marquez et al., 2013; Tang and Hammond, 2013) the mechanistic substrate remains poorly explained.

\section{ANATOMICAL tDCS SPECIFICITY AND THE "SLIDING-SCALE" MODEL}

Anatomical specificity refers to the preferential neuromodulation of targeted brain regions by delivering stimulation current to the targeted area. The number, location, and size of anatomical targets are application specific. For example, the targeted brain region may be a specific cortical area implicated in a task or pathology. Anatomical specificity is achieved only through the control of tDCS electrode dose (defined as electrode montage and current) to guide current to specific brain regions (Peterchev et al., 2011). However, applied without consideration for functional specificity, anatomical specificity is technically and conceptually limited.

Both computational models of current flow in the brain and imaging studies indicate that conventional tDCS methodology using two large sponge pads $(5 \mathrm{~cm} \times 5 \mathrm{~cm})$ positioned on the head disperse current through much of the cortex (Datta et al., 2009; Faria et al., 2011; Antal et al., 2012; Neuling et al., 2012) and even deep brain structures (Dasilva et al., 2012). It is important to distinguish between carefully designed studies that demonstrate dose-specific (e.g., electrode position) outcomes (Fiori et al., 2013; Hauser et al., 2013; Penolazzi et al., 2013), from implications that current flow is limited to one brain target. These studies also typically leverage other forms of functional targeting.

Technology for High-Definition tDCS using arrays of electrodes allows categorical increases in anatomical targeting by increasing the focality of current flow (Datta et al., 2009; Dmochowski et al., 2011), but even so, any brain region is evidently involved in multiple tasks. Which presents the inherent conceptual challenge when relying exclusively on anatomical specificity: how can passing DC current through a multi-tasking complex brain region produce specific functional changes?

In the absence of further sophistication, the goals of tDCS are often described as increasing excitability (near the anode) or decreasing excitability (near the cathode) of the target brain region, with brain function and disease thus reduced to a "slidingscale" of excitability to be adjusted by stimulation (c.f., Rahman et al., 2013). For example, under the sliding-scale concept "anodal tDCS" can enhance the performance of a cognitive task by exciting an implicated brain region. Similarly anodal tDCS is intended to increase left-prefrontal cortex activity in depression 
and enhance rehabilitation around lesions after stroke. Following early evaluation of Transcranial/transcortical polarization in humans and animal models (Bindman et al., 1962; Redfearn et al., 1964; Elbert etal., 1981), influential neurophysiological studies of tDCS (Nitsche and Paulus, 2000) established modulation of experimental evoked potentials [e.g., motor evoked potential responses to transcranial magnetic stimulation (TMS)]. There is significant extrapolation from these experimental findings to behavior and cognition [TMS evoked responses may provide poor evidence for effects on behavior; (Ridding and Rothwell, 2007)]. Moreover, even the direction of this basic modulation of experimentally evoked potentials is highly sensitive to both tDCS dose [intensity (Matsunaga et al., 2004; Dieckhofer et al., 2006; Batsikadze et al., 2013); direction (Chan and Nicholson, 1986; Bikson etal., 2004; Rahman etal., 2013)] and dependent on brain state (Fröhlich and McCormick, 2010; Reato et al., 2010). Animal studies showing anodal/cathodal DCS producing somatic depolarization/hyperpolarizing (Radman et al., 2009) and increase/decrease in firing rate (Purpura and McMurtry, 1965; Reato et al., 2010), are cited to support a sliding-scale concept, however, global changes in firing rate across a brain region implies a non-specific effect (Reato et al., 2013). In summary, it is reasonable to conclude from neurophysiologic studies that tDCS can produce dose-specific changes in brain functions (Nitsche and Paulus, 2000) that can, with careful extrapolation, serve as a basis for behavioral interventions (Kuo et al., 2013). However, relying only on anatomical specificity by guiding current to specific brain regions (and so the "sliding-scale" rationale) remains limited by the complex and divergent functions of any brain region.

Further sophistication in anatomical targeting follows from considering tangential as well as radial inward/outward currents (Dmochowski etal., 2012) as discussed in a separate article in this special issue. Indeed, the assumption of inward ("excitatory") and outward ("inhibitory") current under the anode and cathode, respectively, may be a further over-simplification. Electrophysiological studies in animal models of DC stimulation suggest differential processing of afferent information (Rahman et al., 2013) and that polarity-specific effects invert due to neuronal morphology (Bikson et al., 2004; Kabakov et al., 2012).

\section{ACTIVITY-SELECTIVITY AND TASK-SPECIFIC MODULATION}

Activity-selectivity refers to tDCS preferentially modulating a neuronal network that is already activated, while not modulating separate neuronal network that are inactive. The active neuronal network may be activated for a host of reasons described. The active and inactive networks can in fact overlap in space (e.g., in the same cortical column) such that activity-selectivity does not require physical separation in contrast to anatomical specificity therefore, we refer to activity-selectivity as a form of functional specificity. The active network may represent a subset of neurons and/or a subset of connections (synapses). Because tDCS produces low-intensity electric fields in the brain, "sub-threshold" neuromodulation may reflect changes in ongoing processes (Reato et al., 2010) in contrast to supra-threshold driven firing by TMS. Activity-selectivity thus assumes there is some feature of the active network that makes it preferentially sensitive to modulation by
tDCS compared to other inactive networks. We consider two neurophysiological substrates for this preferred sensitivity: ongoing activity-selectivity and input-selectivity.

Activity-selectivity is based on the assumption that tDCS will preferentially modulate specific forms of ongoing activity. For example, at a cellular level, direct current stimulation (DCS) may enhance plasticity in a given synaptic pathway while stimulated at a preferential frequency $(0.1 \mathrm{~Hz}$ in Fritsch et al., 2010) or consolidate a specific pattern of activity presented during DCS (Morrell, 1961). DCS may preferentially modulate the level of potentiation in the activated pathway (Ranieri et al., 2012). DCS may facilitate long-term potentiation through membrane polarization and removal of $\mathrm{Mg}^{+}$block (Stagg and Nitsche, 2011) but only those pathways activated during DCS (by a task or experimental stimulation) would benefit from this facilitation. DCS may be too weak and/or unspecific in isolation to enhance synaptic efficacy, but may boost ongoing (e.g., Hebbian) plasticity activated by task performance (i.e., modulation of input specific plasticity along an activated synaptic pathway while sparing quiescent synapses). In humans, transcranial electrical stimulation may also preferentially modulate networks with heightened oscillatory activity (Reato et al., 2010) or preferentially change the progression of an active network during memory consolidation or synaptic downscaling (Reato et al., 2013).

At a behavioral level, specific brain activity is often targeted by training in conjunction with tDCS with the goal that this select activity be sensitized to tDCS neuromodulation (and so implicitly other brain functions not active in training may be less so). For example use-dependent modulation and learning of motor skills is modulated by tDCS (Reis and Fritsch, 2011; Madhavan and Shah, 2012). Clinically, tDCS is often applied to enhance the efficacy of rehabilitation or cognitive training (Edwards et al., 2009; Kuo and Nitsche, 2012; Gomez et al., 2013; Leśniak et al., 2013; Ochi et al., 2013), which may further confer functional specificity through activity-selectivity. Clinically when tDCS is applied to subjects at rest, we can speculate that any functional-specificity results from increased sensitivity of pathological network activity to tDCS (e.g., dysfunctional pain or mood regulating networks). It has been speculated that altered network function associated with brain injury (stroke) may alter the susceptibility to tDCS (Olma et al., 2013). Generally, any interaction between brain activity and the efficacy of tDCS modulation (Kim and Ko, 2013; Pirulli et al., 2013) suggests "tDCS can be highly focal when guided by a behavioral task" (Lapenta et al., 2013).

Although the mechanisms may vary, in any case, functional specificity through activity-selectivity presumes the enhanced activity of the network makes it preferentially sensitive to modulation by tDCS. Thus activity-selectivity necessitates an ongoing network process becoming preferentially tuned to influence by DCS compared to the myriad of other ongoing (background) brain functions.

\section{INPUT-SELECTIVITY AND BIAS}

A third form of specificity we define here is input-selectivity, which assumes a neuronal network that is predisposed to serve at least two functions or operate in at least two states such that tDCS can switch the network from one function/state to another: for example 
attentional bias in the prefrontal cortex (Eldar et al., 2013). tDCS would change the state of the system toward a different input bias and thus enhance information processing of a specific stream of information. Input-selectivity may activate endogenous "gating" systems (e.g., gate theory of pain) or bi-stable neuronal states where a non-specific DC signal is able to "switch" a system between complex functions or modes. In contrast to a sliding-scale hypothesis for a stimulated brain region or activity-selectivity affecting a specific ongoing process, input-selectivity implies a regional process is enhanced at the cost of another process - not that input-selectivity results in a zero-sum effect in regards to lasting cognition or behavior outcomes. However, input-selectivity does emphasize the "cost" of acute stimulation. Input-selectivity is also considered a form of functional-specificity since it does not require gross anatomical targeting of current flow. Input-selectivity thus differs conceptually from functional-selectivity (as defined above) in that it does not presuppose co-activation (e.g., by training), and moreover implies that one process may be enhanced at the cost of enhancing another.

Many animal studies that have investigated the modulation of information processing, for example through synaptic efficacy [as opposed to simply membrane polarization and excitability (Chan and Nicholson, 1986; Radman et al., 2007)], have observed that DCS will differentially modulate incoming inputs. We initially showed in hippocampal slice that DCS enhances some and inhibits other afferent inputs (Bikson et al., 2004), a finding verified (Kabakov et al., 2012) and extended to the cortex (Rahman et al., 2013). The cellular origins of bias in favor of selective inputs are twofold. First, although anodal and cathodal tDCS are mistakenly referred to as depolarizing and hyperpolarizing, it is more accurate to describe tDCS as redistributing polarization across the cellular axis, for example one dendritic branch versus another (Fritsch et al., 2010; Rahman et al., 2013). This change in "weights" across the dendrite may provide a cellular substrate to influence the input bias of a network. Second, polarization of afferent axons itself appears to exert pathway specific modulation (Arlotti et al., 2012; Kabakov et al., 2012; Rahman et al., 2013).

Clinically, the concept of input-selectivity can be extended to (selective) attention and working-memory, as well as disease states such as ADHD (Levy, 2004), anxiety, and schizophrenia (Grace, 2000); which are indeed already indications explored for tDCS (Kang et al., 2009; Faber et al., 2012; Demirtas-Tatlidede et al.,

\section{REFERENCES}

Antal, A., Bikson, M., Datta, A., Lafon, B., Dechent, P., Parra, L. C., et al. (2012). Imaging artifacts induced by electrical stimulation during conventional fMRI of the brain. Neuroimage doi: 10.1016/j.neuroimage.2012.10.026 [Epub ahead of print].

Arlotti, M., Rahman, A., Minhas, P., and Bikson, M. (2012). Axon terminal polarization induced by weak uniform DC electric fields: a modeling study. Conf. Proc. IEEE Eng. Med. Biol. Soc. 2012, 4575-
4578. doi: 10.1109/EMBC.2012 6346985

Batsikadze, G., Moliadze, V., Paulus, W., Kuo, M. F., and Nitsche, M. A. (2013). Partially non-linear stimulation intensity-dependent effects of direct current stimulation on motor cortex excitability in humans. J. Physiol. 591, 1987-2000. doi: 10.1113/jphysiol.2012.249730

Bikson, M., Inoue, M., Akiyama, H., Deans, J. K., Fox, J. E., Miyakawa, H., et al. (2004). Effects of uniform extracellular DC electric fields on excitability in rat hippocampal

2013; Shiozawa et al., 2013). The prefrontal cortex, an anatomical target for several indications including depression (Loo et al., 2012), is indeed implicated in executive function and differentiating among (conflicting) inputs. The concept of bias-selection is consistent with stimulation inhibiting some functions while enhancing others within any given region (Iuculano and Kadosh, 2013; Tang and Hammond, 2013) as well as modulation of relative value judgments (Vanderhasselt et al., 2013; Votinov et al., 2013), which can be considered as a form of weighting inputs so specific outcomes can be biased.

Future studies on the actions of input-selectivity using tDCS can explore applications of multimodal imaging technologies, including magnetic resonance spectroscopy (MRS), functional magnetic resonance imaging (fMRI), magneto-encephalogram (MEG), and electro-encephalogram (EEG), to further establish the different functional brain-states of a cortical region activated during tDCS (Soekadar et al., 2013). The topic of multimodal imaging in tDCS is discussed further in another article in this special issue of Frontiers in Human Neuroscience (Hunter et al., 2013).

\section{OUTLOOK FOR tDCS}

Anatomical specificity and functional specificity, through either ongoing activity-selectivity or input-selectivity, are not exclusive and may potentially be leveraged together in the development of rational tDCS protocols. In general, we propose that understanding the basis for tDCS selectivity is essential. Although we have focused our discussion to tDCS, the approaches described here would apply to other brain stimulation techniques including DBS, VNS, TMS, tRNS, and tACS (discussed further in another article in this special issue, Reato et al., 2013) as well as ultrasound and light based approaches. But the diversity of applications already investigated for tDCS, including increasing dosage (e.g., weeks of sessions), broader populations (e.g., children), suggests a need to address the basis of specificity to be especially acute. Both timedependent and homeostatic effects (Penolazzi et al., 2013; Peters et al., 2013) increase the subtlety in tDCS protocol design that may require understanding origins of specificity.

\section{ACKNOWLEDGMENT}

The authors would like to thank Fred Callahan for valuable insights. This work is supported by The Wallace H. Coulter Foundation, NIH grant RO1 MH-092926, NSF, and the DOD/US Air Force.

slices in vitro. J. Physiol. 557, 175-190. doi: 10.1113/jphysiol.2003. 055772

Bindman, L. J., Lippold, O. C., and Redfearn, J. W. (1962). Long-lasting changes in the level of the electrical activity of the cerebral cortex produced by polarizing currents. Nature 196, 584-585. doi: 10.1038/196584a0 Brunoni, A. R., Nitsche, M. A., Bolognini, N., Bikson, M., Wagner, T., Merabet, L., et al. (2012). Clinical research with transcranial direct current stimulation (tDCS): challenges and future directions.
Brain Stimul. 5, 175-195. doi: 10.1016/j.brs.2011.03.002

Cano, T., Morales-Quezada, J. L., Bikson, M., and Fregni, F. (2013). Methods to focalize noninvasive electrical brain stimulation: principles and future clinical development for the treatment of pain. Expert Rev. Neurother. 13, 465-467. doi: 10.1586/ern.13.41

Chan, C. Y., and Nicholson, C. (1986). Modulation by applied electric fields of Purkinje and stellate cell activity in the isolated turtle cerebellum. J. Physiol. 371, 89-114. 
Dasilva, A. F., Mendonca, M. E., Zaghi, S., Lopes, M., Dossantos, M. F., Spierings, E. L., et al. (2012). tDCS-induced analgesia and electrical fields in pain-related neural networks in chronic migraine. Headache 52, 1283-1295. doi: 10.1111/j.15264610.2012.02141.x

Datta, A., Bansal, V., Diaz, J., Patel, J., Reato, D., and Bikson, M. (2009) Gyri-precise head model of transcranial direct current stimulation: improved spatial focality using a ring electrode versus conventional rectangular pad. Brain Stimul. 2, 201-207. doi: 10.1016/j.brs.2009.03.005

Demirtas-Tatlidede, A., VahabzadehHagh, A. M., and Pascual-Leone, A. (2013). Can noninvasive brain stimulation enhance cognition in neuropsychiatric disorders? Neuropharmacology 64, 566-578. doi: 10.1016/j.neuropharm.2012.06.020

Dieckhofer, A., Waberski, T. D., Nitsche, M., Paulus, W., Buchner, H., and Gobbele, R. (2006). Transcranial direct current stimulation applied over the somatosensory cortex - differential effect on low and high frequency SEPs. Clin. Neurophysiol. 117, 2221-2227. doi: 10.1016/j.clinph.2006.07.136

Dmochowski, J. P., Bikson, M., Datta, A., Richardson, J., Fridriksson, J., and Parra, L. C. (2012). On the role of electric field orientation in optimal design of transcranial current stimulation. Conf. Proc. IEEE Eng. Med. Biol. Soc. 2012, 6426-6429. doi: 10.1109/EMBC.2012.6347465

Dmochowski, J. P., Datta, A., Bikson, M., Su, Y., and Parra, L. C. (2011). Optimized multi-electrode stimulation increases focality and intensity at target. J. Neural Eng. 8, 046011. doi: 10.1088/1741-2560/8/4/046011

Edwards, D. J., Krebs, H. I., Rykman, A., Zipse, J., Thickbroom, G. W., Mastaglia, F. L., et al. (2009). Raised corticomotor excitability of M1 forearm area following anodal tDCS is sustained during robotic wrist therapy in chronic stroke. Restor. Neurol. Neurosci. 27, 199-207. doi: 10.3233/RNN-2009-0470

Elbert, T., Lutzenberger, W., Rockstroh, B., and Birbaumer, N. (1981). The influence of low-level transcortical DC-currents on response speed in humans. Int. J. Neurosci. 14, 101-114. doi: 10.3109/00207458108985821

Eldar, E., Cohen, J. D., and Niv, Y. (2013). The effects of neural gain on attention and learning. Nat. Neurosci. 16, 1146-1153. doi: 10.1038/nn.3428

Faber, M., Vanneste, S., Fregni, F., and De Ridder, D. (2012). Top down prefrontal affective modulation of tinnitus with multiple sessions of tDCS of dorsolateral prefrontal cortex. Brain Stimul. 5, 492-498. doi: 10.1016/j.brs.2011.09.003

Faria, P., Hallett, M., and Miranda, P. C. (2011). A finite element analysis of the effect of electrode area and inter-electrode distance on the spatial distribution of the current density in tDCS. J. Neural Eng. 8, 066017. doi: 10.1088/1741-2560/8/6/066017

Fiori, V., Cipollari, S., Di Paola, M., Razzano, C., Caltagirone, C., and Marangolo, P. (2013). tDCS stimulation segregates words in the brain: evidence from aphasia. Front. Hum. Neurosci. 7:269. doi: 10.3389/fnhum.2013.00269

Fritsch, B., Reis, J., Martinowich, K., Schambra, H. M., Ji, Y., Cohen, L. G., et al. (2010). Direct current stimulation promotes BDNF-dependent synaptic plasticity: potential implications for motor learning. Neuron 66 , 198-204. doi: 10.1016/j.neuron.2010. 03.035

Fröhlich, F., and McCormick, D. A. (2010). Endogenous electric fields may guide neocortical network activity. Neuron 67, 129-143. doi: 10.1016/j.neuron.2010.06.005

Gomez, P. S. A., Faraji, J., Metz, G. A., Tatsuno, M., and Luczak, A. (2013). Transcranial direct current stimulation in stroke rehabilitation: a review of recent advancements. Stroke Res. Treat. 2013, 170256. doi: 10.1155/2013/170256

Grace, A. A. (2000). Gating of information flow within the limbic system and the pathophysiology of schizophrenia. Brain Res. Brain Res. Rev. 31, 330-341. doi: 10.1016/S0165-0173(99)00049-1

Hauser, T. U., Rotzer, S., Grabner, R. H., Merillat, S., and Jancke, L. (2013). Enhancing performance in numerical magnitude processing and mental arithmetic using transcranial direct current stimulation (tDCS). Front. Hum. Neurosci. 7:244. doi: 10.3389/fnhum.2013.00244

Hunter, M., Coffman, B., Trumbo, M., and Clark, V. (2013). Tracking the neuroplastic changes associated with transcranial direct current stimulation: a push for multimodal imaging. Front. Hum. Neurosci. 7:495. doi: 10.3389/fnhum.2013.00495

Iuculano, T., and Kadosh, R. C. (2013). The mental cost of cognitive enhancement. $J$. Neurosci. 33, 4482-4486. doi: 10.1523/JNEUROSCI.4927-12.2013

Kabakov, A. Y., Muller, P. A., PascualLeone, A., Jensen, F. E., and Rotenberg, A. (2012). Contribution of axonal orientation to pathway- dependent modulation of excitatory transmission by direct current stimulation in isolated rat hippocampus J. Neurophysiol. 107, 1881-1889. doi: 10.1152/jn.00715.2011

Kang, E. K., Baek, M. J., Kim, S., and Paik, N. J. (2009). Non-invasive cortical stimulation improves poststroke attention decline. Restor. Neurol. Neurosci. 27, 645-650. doi: 10.3233/RNN-2009-0514

Kim, G. W., and Ko, M. H. (2013). Facilitation of corticospinal tract excitability by transcranial direct current stimulation combined with voluntary grip exercise. $\mathrm{Neu}$ rosci. Lett. 548, 181-184. doi: 10.1016/j.neulet.2013.05.037

Kuo, M. F., and Nitsche, M. A. (2012). Effects of transcranial electrical stimulation on cognition. Clin. EEG Neurosci. 43, 192-199. doi: $10.1177 / 1550059412444975$

Kuo, M. F., Paulus, W., and Nitsche, M. A. (2013). Therapeutic effects of non-invasive brain stimulation with direct currents (tDCS) in neuropsychiatric diseases. Neuroimage doi: 10.1016/j.neuroimage.2013.05.117 [Epub ahead of print]

Lapenta, O. M., Minati, L., Fregni, F., and Boggio, P. S. (2013). Je pense donc je fais: transcranial direct current stimulation modulates brain oscillations associated with motor imagery and movement observation. Front. Hum. Neurosci. 7:256. doi: 10.3389/fnhum.2013.00256

Leśniak, M., Polanowska, K., Seniów, J., Członkowska, A. (2013). Effects of repeated anodal tDCS coupled with cognitive training for patients with severe traumatic brain injury: a pilot randomized controlled trial. J. Head Trauma Rehabil. doi: 10.1097/HTR.0b013e318292a4c2 [Epub ahead of print].

Levy, F. (2004). Synaptic gating and ADHD: a biological theory of comorbidity of ADHD and anxiety. Neuropsychopharmacology 29, 15891596. doi: 10.1038/sj.npp.1300469

Loo, C. K., Alonzo, A., Martin, D., Mitchell, P. B., Galvez, V., and Sachdev, P. (2012). Transcranial direct current stimulation for depression: 3-week, randomised, sham-controlled trial. Br. J. Psychiatry 200, 52-59. doi: 10.1192/bjp.bp.111.097634

Madhavan, S., and Shah, B. (2012). Enhancing motor skill learning with transcranial direct current stimulation - a concise review with applications to stroke. Front. Psychiatry 3:66. doi: 10.3389/fpsyt.2012.00066

Matsunaga, K., Nitsche, M. A., Tsuji, S., and Rothwell, J. C. (2004). Effect of transcranial DC sensorimotor cortex stimulation on somatosensory evoked potentials in humans. Clin. Neurophysiol. 115, 456-460. doi: 10.1016/S1388-2457(03)00362-6

Medina, J., Beauvais, J., Datta, A., Bikson, M., Coslett, H. B., and Hamilton, R. H. (2013). Transcranial direct current stimulation accelerates allocentric target detection. Brain Stimul. 6, 433-439. doi: 10.1016/j.brs.2012.05.008

Morrell, F. (1961). Effect of anodal polarization on the firing pattern of single cortical cells. Ann. N. Y. Acad. Sci. 92, 860-876. doi: 10.1111/j.17496632.1961.tb40962.x

Neuling, T., Wagner, S., Wolters, C. H., Zaehle, T., and Herrmann, C. S. (2012). Finite-element model predicts current density distribution for clinical applications of tDCS and tACS. Front. Psychiatry 3:83. doi: 10.3389/fpsyt.2012.00083

Nitsche, M. A., and Paulus, W. (2000). Excitability changes induced in the human motor cortex by weak transcranial direct current stimulation. J. Physiol. 527(Pt 3), 633-639. doi: 10.1111/j.1469-7793.2000.t01-100633.x

Ochi, M., Saeki, S., Oda, T., Matsushima, Y., and Hachisuka, K. (2013). Effects of anodal and cathodal transcranial direct current stimulation combined with robotic therapy on severely affected arms in chronic stroke patients. J. Rehabil. Med. 45, 137-140. doi: 10.2340/165019771099

Olma, M. C., Dargie, R. A., Behrens, J. R., Kraft, A., Irlbacher, K., Fahle, M., et al. (2013). Longterm effects of serial anodal tDCS on motion perception in subjects with occipital stroke measured in the unaffected visual hemifield. Front. Hum. Neurosci. 7:314. doi: 10.3389/fnhum.2013.00314

Penolazzi, B., Pastore, M., and Mondini, S. (2013). Electrode montage dependent effects of transcranial direct current stimulation on semantic fluency. Behav. Brain Res. 248, 129-135. doi: 10.1016/j.bbr.2013.04.007

Peterchev, A. V., Wagner, T. A., Miranda, P. C., Nitsche, M. A., Paulus, W., Lisanby, S. H., et al. (2011). Fundamentals of transcranial electric and magnetic stimulation dose: definition, selection, and reporting practices. Brain Stimul. 5, 435-453. doi: 10.1016/j.brs.2011.10.001

Peters, M. A., Thompson, B., Merabet, L. B., Wu, A. D., and Shams, L. (2013). Anodal tDCS to V1 blocks visual perceptual learning consolidation. Neuropsychologia 51, 1234-1239. 
doi: $\quad$ 10.1016/j.neuropsychologia. 2013.03.013

Pirulli, C., Fertonani, A., and Miniussi, C. (2013). The role of timing in the induction of neuromodulation in perceptual learning by transcranial electric stimulation. Brain Stimul. 6, 683-689. doi: 10.1016/j.brs.2012.12.005

Purpura, D. P., and McMurtry, J. G. (1965). Intracellular activities and evoked potential changes during polarization of motor cortex. $J$. Neurophysiol. 28, 166-185.

Radman, T., Ramos, R. L., Brumberg, J. C., and Bikson, M. (2009). Role of cortical cell type and morphology in subthreshold and suprathreshold uniform electric field stimulation in vitro. Brain Stimul. 2, 215-228. doi: 10.1016/j.brs.2009.03.007

Radman, T., Su, Y. Z., An, J. H., Parra, L. C., and Bikson, M. (2007). Spike timing amplifies the effect of electric fields on neurons: implications for endogenous field effects. J. Neurosci. 27, 3030-3036. doi: 10.1523/JNEUROSCI.0095-07.2007

Rahman, A., Reato, D., Arlotti, M., Gasca, F., Datta, A., Parra, L. C., et al. (2013). Cellular effects of acute direct current stimulation: somatic and synaptic terminal effects. J. Physiol. 591(Pt 10), 2563-2578. doi: 10.1113/jphysiol.2012.247171

Ranieri, F., Podda, M. V., Riccardi, E. Frisullo, G., Dileone, M., Profice, P., et al. (2012). Modulation of Ltp at rat hippocampal Ca3-Ca1 synapses by direct current stimulation. $J$. Neurophysiol. 107, 1868-1880. doi: 10.1152/jn.00319.2011

Reato, D., Gasca, F., Datta, A., Bikson, M., Marshall, L., and Parra, L. C. (2013). Transcranial electrical stimulation accelerates human sleep homeostasis. PLoS Comput. Biol. 9:e1002898. doi: 10.1371/journal. pcbi. 1002898

Reato, D., Rahman, A., Bikson, M. and Parra, L. C. (2010). Lowintensity electrical stimulation affects network dynamics by modulating population rate and spike timing. J. Neurosci. 30, 15067-15079. doi: 10.1523/JNEUROSCI.2059-10.2010

Redfearn, J. W., Lippold, O. C. and Costain, R. (1964). A preliminary account of the clinical effects of polarizing the brain in certain psychiatric disorders. $\mathrm{Br}$. J. Psychiatry 110, 773-785. doi: 10.1192/bjp.110.469.773

Reis, J., and Fritsch, B. (2011). Modulation of motor performance and motor learning by transcranial direct current stimulation. Curr. Opin. Neurol. 24, 590 596. doi: 10.1097/WCO.0b013e $32834 \mathrm{c} 3 \mathrm{db} 0$

Ridding, M. C., and Rothwell, J. C. (2007). Is there a future for therapeutic use of transcranial magnetic stimulation? Nat. Rev. Neurosci. 8, 559-567. doi: 10.1038/nrn2169

Ruffini, G., Wendling, F., Merlet, I., Molaee-Ardekani, B., Mekkonen, A., Salvador, R., et al. (2012). Transcranial current brain stimulation (tCS): models and technologies. IEEE Trans. Neural. Syst. Rehabil. Eng. 21, 333-345. doi: 10.1109/TNSRE.2012.2200046

Saucedo Marquez, C. M., Zhang, X., Swinnen, S. P., Meesen, R., and Wenderoth, N. (2013). Task-specific effect of transcranial direct current stimulation on motor learning. Front. Hum. Neurosci. 7:333. doi: 10.3389/fnhum.2013.00333
Shiozawa, P., Da Silva, M. E., Cordeiro, Q., Fregni, F., and Brunoni, A. R. (2013). Transcranial direct current stimulation (tDCS) for the treatment of persistent visual and auditory hallucinations in schizophrenia: a case study. Brain Stimul. 6, 831-833. doi: 10.1016/j.brs.2013.03.003

Soekadar, S. R., Witkowski, M., Cossio, E. G., Birbaumer, N., Robin son, S. E., and Cohen, L. G. (2013). In vivo assessment of human brain oscillations during application of transcranial electric currents. Nat. Commun. 4, 2032. doi: 10.1038/ ncomms 3032

Stagg, C. J., and Nitsche, M. A. (2011). Physiological basis of transcranial direct current stimulation. Neuroscientist 17 37-53. doi: 10.1177/1073858410 386614

Tang, M. F., and Hammond, G. R. (2013). Anodal transcranial direct current stimulation over auditory cortex degrades frequency discrimination by affecting temporal, but not place, coding. Eur. J. Neurosci. 38, 2802-2811. doi: 10.1111/ejn. 12280

Turkeltaub, P. E., Benson, J., Hamilton, R. H., Datta, A., Bikson, M., and Coslett, H. B. (2012). Left lateralizing transcranial direct current stimulation improves reading efficiency. Brain Stimul. 5, 201-207 doi: 10.1016/j.brs.2011.04.002

Vanderhasselt, M. A., De Raedt, R., Brunoni, A. R., Campanha, C., Baeken, C., Remue, J., et al. (2013). tDCS over the left prefrontal cortex enhances cognitive control for positive affective stimuli. PLoS ONE 8:e62219. doi: 10.1371/journal.pone.0062219
Votinov, M., Aso, T., Koganemaru, S., Fukuyama, H., and Mima, T. (2013). Transcranial direct current stimulation changes human endowment effect. Neurosci. Res. 76, 251-256. doi: 10.1016/j.neures.2013. 05.007

Zimerman, M., Nitsch, M., Giraux, P., Gerloff, C., Cohen, L. G., and Hummel, F. C. (2013). Neuroenhancement of the aging brain: restoring skill acquisition in old subjects. Ann. Neurol. 73, 10-15. doi: 10.1002/ana. 23761

Conflict of Interest Statement: The authors declare that the research was conducted in the absence of any commercial or financial relationships that could be construed as a potential conflict of interest.

Received: 08 July 2013; accepted: 30 September 2013; published online: 21 October 2013.

Citation: Bikson $M$ and Rahman $A$ (2013) Origins of specificity during tDCS: anatomical, activity-selective, and inputbias mechanisms. Front. Hum. Neurosci. 7:688. doi: 10.3389/fnhum.2013.00688

This article was submitted to the journal Frontiers in Human Neuroscience. Copyright (c) 2013 Bikson and Rahman. This is an open-access article distributed under the terms of the Creative Commons Attribution License (CC BY). The use, distribution or reproduction in other forums is permitted, provided the original author(s) or licensor are credited and that the original publication in this journal is cited, in accordance with accepted academic practice. No use, distribution or reproduction is permitted which does not comply with these terms 